CAHIERS DE

NARRATOLOGIE

\section{Cahiers de Narratologie}

Analyse et théorie narratives

27 | 2014

Les bifurcations du récit interactif: continuité ou rupture?

\title{
Où va l'intrigue ? Réflexions autour de quelques récits fortement interactifs
}

\section{Nicolas Szilas}

\section{(2) OpenEdition \\ Journals}

Electronic version

URL: http://journals.openedition.org/narratologie/7065

DOI: $10.4000 /$ narratologie.7065

ISSN: 1765-307X

Publisher

LIRCES

\section{Electronic reference}

Nicolas Szilas, "Où va l'intrigue? Réflexions autour de quelques récits fortement interactifs », Cahiers de Narratologie [Online], 27 | 2014, Online since 19 December 2014, connection on 19 April 2019. URL: http://journals.openedition.org/narratologie/7065; DOI : 10.4000/narratologie.7065

This text was automatically generated on 19 April 2019

Article L.111-1 du Code de la propriété intellectuelle. 


\title{
Où va l'intrigue? Réflexions autour de quelques récits fortement interactifs
}

\author{
Nicolas Szilas
}

\section{Remerciements}

Ces travaux de recherche n'auraient pas pu voir le jour sans le soutien du Fonds National de la Recherche Suisse, via le projet « TBI-SIM » (co-dirigé par Jean Dumas et Nicolas Szilas).

\section{Emergence d'une nouvelle forme narrative : le récit fortement interactif}

1 Nous nous intéresserons dans cette contribution à ce que nous appelons le récit fortement interactif. Pensé dans les années $1980^{1}{ }^{2}$, celui-ci fait l'objet de recherches informatiques plus étendues depuis la fin des années 1990. L'objectif de ces recherches, pour ne pas dire l'idéal, est de produire des récits dans lesquels le spectateur contrôle fortement le déroulement de l'histoire. Il s'agit de donner au lecteur une "agency » narrative, comme la définit Janet Murray dans son livre au titre emblématique, « Hamlet in the Holodeck » : la satisfaction de pouvoir exercer de véritables actions et d'observer l'effet de nos choix et décisions ${ }^{3}$. Il a beaucoup été écrit sur les potentialités d'un tel programme, notamment en terme de bouleversement de l'intrigue et du rapport entre le spectateur, l'histoire, la machine et l'auteur. Mais qu'en est-il en réalité, dans le concret des récits fortement interactifs? Pendant longtemps, le domaine n'a pas réellement dépassé le stade du prototype technique, insuffisamment développé pour laisser entrevoir les œuvres promises par les acteurs du domaine. Ainsi, en 2006, on pouvait parler à son propos de " chimère » ou de "graal ", tant le discours sur le récit interactif tendait à écarter les œuvres existantes, sans pouvoir pour autant en proposer une seule ${ }^{4}$. De manière corollaire se pose la question de la définition : pourquoi tel ou tel jeu vidéo n'est pas un récit fortement interactif auquel aspirent les chercheurs? La définition du champ est malaisée : si on le définit en terme de liberté offerte au joueur, donc de nombre de choix offerts, on pourra toujours objecter qu'un récit sous forme de simple graphe, 
s'appuyant donc sur des principes techniques bien connus, peut satisfaire cette définition, pour peu qu'un auteur prenne le temps d'écrire toutes les variantes possibles. $C^{\prime}$ est pourquoi nous proposerons une définition plus technique d'un récit fortement interactif. Celui-ci est une œuvre informatique qui :

- met en scène des événements qui sont le résultat d'un calcul, tant au niveau de leur occurrence (si ça arrive et quand ça arrive), leur contenu (acte narratif) que leur formulation;

- donne à l'utilisateur le rôle d'un personnage principal de l'histoire ;

- induit chez l'utilisateur une expérience qu'il qualifie de narrative.

5 Depuis peu quelques récits fortement interactifs ont pu voir le jour, allant au delà du stade de simple prototype technique, c'est-à-dire des récits entiers, accessibles, et porteurs d'une intention artistique. Ainsi, pour étayer notre propos, nous nous appuierons sur trois oeuvres (voir Figure 1): Façade ${ }^{5}$, The Unwelcome Proposal ${ }^{6}$, et Nothing For Dinner ${ }^{7}$, ce dernier ayant été écrit et réalisé au sein de notre laboratoire. Façade, sorti en 2006, est l'œuvre emblématique du domaine. L'utilisateur est plongé dans une scène de couple dont l'issue dépend de son intervention en tant que vieil ami du couple. Agissant essentiellement via des textes courts tapés librement au clavier, plus ou moins bien compris par la machine, l'utilisateur dialogue avec l'un ou l'autre des membres du couple, qui réagissent à ses propos. Les personnages, représentés dans un environnement 3D, s'expriment via des enregistrements audio d'acteurs et une animation faciale expressive (voir Figure 1.a). The Unwelcome Proposal, réalisée à l'aide de la plateforme Versu, propose une adaptation d'une scène de "Pride and Prejudice» de Jane Austen. L'utilisateur peut incarner un des personnages principaux et se voit proposer, sous forme textuelle, des choix qui vont modifier le déroulement de la scène. Proche d'un « livre dont vous êtes le héros ", cette œuvre propose néanmoins une liberté d'action inédite. Enfin, Nothing For Dinner (ci-après NFD), réalisé dans notre laboratoire et sur lequel nous appuierons donc l'essentiel de notre article, est un jeu pédagogique visant à aider des adolescents dont l'un des parents souffre d'un traumatisme crânien. Le joueur incarne ainsi un adolescent dont le père, atteint d'un traumatisme crânien, perturbe le quotidien familial par ses remarques, attitudes et actions. Le joueur a pour « mission » de préparer le repas du soir, tout en s'efforçant de gérer le comportement difficile du père, entouré aussi d'une jeune sœur et de la grand-mère. Navigant dans un environnement 3D, le joueur sélectionne une action parmi la vingtaine d'actions proposées en permanence, réparties entre les différents personnages et objets de l'œuvre.

Ces œuvres, malgré leurs différences, partagent un point commun : elles sont le résultat d'un compromis entre l'idéal aujourd'hui encore inaccessible de la " narrative agency » et un certaine linéarité. Ainsi, Façade parvient à mettre en scène une «local agency », au détriment d'une "global agency ${ }^{8}$ ", l'utilisateur n'ayant pour impact que le déclenchement de scènes pré-écrites, même si elles sont variables et interruptibles. Dans The Unwelcome Proposal, une interaction riche avec les personnages est possible, via une technologie à base "d'agents intelligents", mais elle se combine avec une progression multi-lineaire de l'histoire, sous forme de graphe, pour faire progresser l'histoire. Dans Nothing For Dinner enfin, le grand nombre de choix possibles offert par la technologie se combine avec un fil narratif conducteur plus ou moins pré-écrit, à savoir le déroulement de la préparation du repas. Si aucune de ces œuvres n'atteint donc l'idéal du récit interactif, elles nous permettent néanmoins de commencer à évaluer les nouveaux 
rapports qui peuvent se mettre en place entre le lecteur actif et l'intrigue. Ces œuvres, complexes, ne peuvent se réduire à un seul phénomène narratif. Notamment, nous avons pu observer qu'elles superposent plusieurs types d'intrigues, selon les mécanismes qui les ont suscitées. Nous proposons donc dans ce qui suit une typologie des intrigues du récit fortement interactif. Etayée par un nombre très faible d'œuvres, cette typologie demeure exploratoire. Nous espérons néanmoins qu'elle pourra servir de grille d'analyse et d'aide à la conception pour les récits interactifs à venir.

Figure 1 : Trois rares exemples de récit fortement interactifs

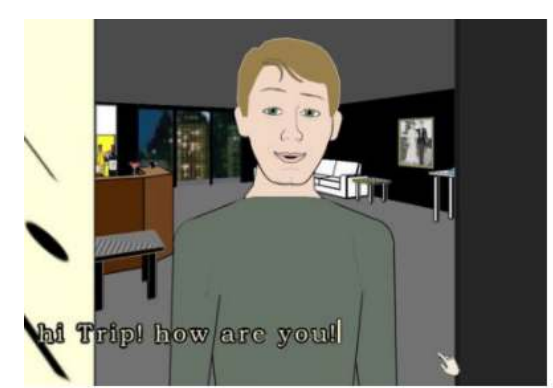

a) Façade

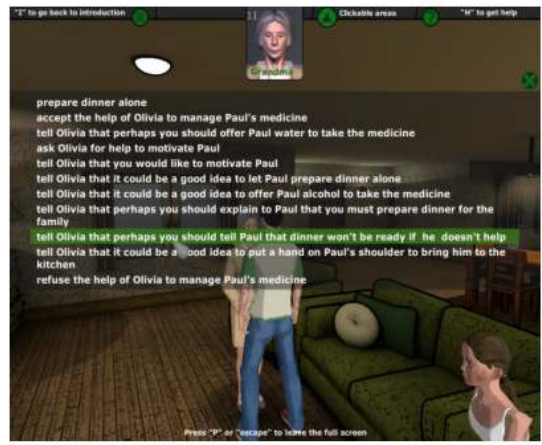

c) Nothing For Dinner

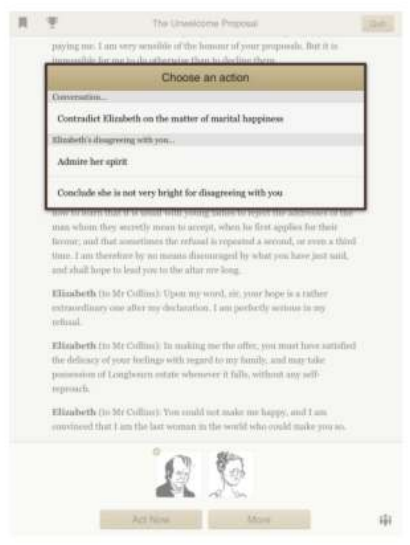

b) The Unwelcome Proposal

\section{Proposition d'une typologie des intrigues du récit fortement interactif}

\section{Les intrigues écrites}

7 Nous proposons tout d'abord de distinguer les intrigues écrites des intrigues que nous appelons résultantes. Les intrigues écrites sont celles qui sont pensées par l'auteur puis "écrites» par l'auteur. Cette écriture est radicalement différente de l'écriture traditionnelle d'un roman ou même d'un script, dans la mesure où elle manipule un matériau formalisé et abstrait ${ }^{9}$. Néanmoins, cette écriture s'appuie sur des relations d'ordre entre des événements prédéfinis (à quelques variantes près), et l'auteur est en mesure d'identifier ces intrigues lors d'une session de jeu. Les intrigues écrites se composent elles-mêmes de deux types d'intrigues: l'intrigue principale et l'intrigue secondaire.

8 L'intrigue principale est le squelette du récit. A la fois unique (un récit n'en possède qu'une) et obligatoire (aucune session de jeu ne pourra passer outre cette intrigue), elle 
s'apparente à l'intrigue telle qu'on la connaît dans les média linéaires. Elle s'en distingue néanmoins par quelques éléments de variabilité :

9 - elle peut se présenter de manière linéaire mais aussi multi-linéaire, sous la forme d'un arbre ;

10 - elle est conçue pour pouvoir être "distendue ", c'est-à-dire que d'autres éléments, pouvant faire partie d'une autre intrigue, peuvent s'insérer entre les événements de l'intrigue principale. Ces inserts sont dynamiquement calculés par la machine et ne peuvent donc pas être totalement maîtrisés par l'auteur.

11 - la représentation médiatique de cette intrigue est susceptible de variation. Pour l'image par exemple, l'angle de prise de vue en 3D d'un même événement varie entre deux sessions. Concernant le texte, la formulation précise peut aussi varier, car les événements sont souvent codés à un niveau abstrait et ce qu'on dénomme en linguistique informatique leur réalisation de surface introduit une variation.

12 Voici pour exemple l'intrigue principale de NFD, résumée ici en langue naturelle, par opposition au langage formel et abstrait effectivement utilisé par l'auteur dans le système :

13 Frank, un adolescent, doit préparer le repas à la maison, alors que sa mère est sortie. Il doit le faire avec son père Paul, qui souffre d'un traumatisme crânien. Il faudra motiver Paul, couper les légumes avec lui, faire cuire la soupe, amener Paul à table, appeler tout le monde à table et enfin s'assoir. Fin.

14 Toutes les étapes de cette intrigue sont des passages obligés du récit, contrairement aux autres événements, qui surviennent ou non selon les sessions. Pour autant, cette intrigue ne constituera dans aucune session la totalité de l'intrigue, car d'autres événements viendront s'insérer. C'est pour cela que nous utilisions plus haut le terme de squelette d'histoire.

Dans Façade, l'intrigue principale est plus difficile à décrire, étant donnée la variabilité. L'histoire se résume par une succession de scènes dont la tension va en croissant, cette escalade menant à l'une des quelques fins possibles, certaines conduisant à la rupture du couple, d'autres, plus rares, à sa réconciliation.

16 L'intrigue principale représente d'une certaine manière le contre-objectif du récit fortement interactif. En effet, dans l'idéal de ce dernier, il n'existe pas d'intrigue principale écrite, l'intrigue devant résulter avant tout de l'action de l'utilisateur. Dans le concret des œuvres observées, l'intrigue principale écrite est présente, et c'est précisément à ce niveau que se situe le compromis évoqué plus haut.

17 Les intrigues écrites secondaires viennent étoffer l'intrigue principale. Les intrigues secondaires ne sont ni uniques ni obligatoires. Une même intrigue secondaire peut-même être présente deux fois dans le récit, sachant que, selon les mêmes principes de variabilité que pour l'intrigue principale, ce ne sera pas exactement la même séquence d'événements. L'intrigue écrite secondaire s'apparente donc à son équivalent en écriture classique, plus particulièrement filmique ${ }^{10}$, à cette importante différence près que la manière dont l'intrigue secondaire s'entremêle avec l'intrigue principale est peu contrôlée par l'auteur. Voici un premier exemple d'intrigue secondaire dans NFD :

18 Julia va arriver pour récupérer son livre. Il faudra l'accueillir, en lui expliquant ou non la situation du père. Ce dernier demandera qui elle est, il faudra lui répondre ou laisser Julia le faire. Il posera des questions embarrassantes, qu'il faudra ignorer (si l'on n'a pas 
expliqué la situation à Julia, elle s'en offusquera, il faudra alors la lui expliquer). On pourra alors lui donner le livre et Julia partira.

19 Cette intrigue secondaire s'avère, dans cet exemple, aussi longue que l'intrigue principale, et un peu plus complexe dans ses déclenchements conditionnels. Secondaire car optionnelle, cette intrigue peut par ailleurs ne pas être résolue quand l'intrigue principale se termine et clôt le récit. l'écriture/conception du récit: un intrigue résultante contient des événements dont l'enchaînement n'a pas été écrit par l'auteur, mais calculé par la machine, selon des règles et mécanismes plus complexes et abstraits. Nous distinguons ensuite deux types d'intrigues résultantes : les intrigues contrôlées et les intrigues coïncidentes.

Contrairement aux intrigues écrites, les intrigues résultantes sont le résultat d'un calcul. Dans le cas le plus notable, l'auteur lui-même n'a pas prévu ces intrigues, et ainsi se surprend lui-même en jouant à son propre récit interactif, phénomène qui existe aussi dans les jeux vidéo. Cependant, on peut difficilement faire de cette caractéristique un critère définitoire étant donné le caractère subjectif qui lui est attaché: comment mesurer cet état de surprise chez l'auteur? Et si les auteurs sont multiples? A partir de quand parle-t-on de surprise, étant donné que d'autres critères de variabilité existent aussi dans les intrigues écrites? De plus, une intrigue ou une partie d'intrigue peut être non prévue par l'auteur, mais pour autant prévisible par ce dernier, s'il a su anticiper les interactions de différentes mécaniques; dans ce cas, l'auteur ne sera pas surpris. Nous souhaitons considérer ce type de cas comme une intrigue résultante.

Par conséquent, il convient de caractériser l'intrigue résultante uniquement du côté de

Dans les intrigues contrôlées, l'arrangement des événements de l'intrigue a été calculé par le système, selon des règles conçues pour constituer une intrigue, au sens large, c'està-dire selon des principes d'ordre narratif. Si, par exemple, un système de récit interactif déclenche un événement pour faire échouer le joueur, car il a calculé que cet échec était important pour la suite du récit, alors il s'agit d'une intrigue calculée. On trouve par exemple ce mécanisme dans le système Mimesis, sous l'appellation de « intervention »: le système fait échouer le tir du joueur, car la mort du personnage visé mettrait en péril la suite de l'intrigue ${ }^{11}$. Dans NFD, on observe fréquemment des intrigues contrôlées. Prenons cette séquence d'actions, retranscrite textuellement pour les besoins de l'article : 

peut apparaitre assez naturelle dans l'exemple ci-dessus, on trouvera des exemples
d'interprétation beaucoup plus personnelle, phénomène que l'on retrouve dans les récits 
classiques. On notera aussi que la distinction entre intrique coïncidente et intrigue écrite ou calculée ne peut se faire sans la connaissance de la manière dont fonctionne le moteur narratif, et ne peut donc être discernée par l'utilisateur non initié à ce fonctionnement. En d'autres termes, pour l'utilisateur naïf, les types d'intrigues que nous avons décrits se superposent sans distinction claire.

Cette discussion sur les intrigues résultantes n'a pas utilisé les termes «émergence » ou «émergent(e)». La raison en est que le terme est chargé de multiples interprétations, selon qu'on parle du concept d'émergence en intelligence artificielle, du concept de "narrative emergence " dans le domaine du récit interactif, ou encore du concept de « emergent gameplay » utilisé dans le milieu du jeu vidéo, y compris par les joueurs. Pour autant, le concept d'intrigue résultante est lié à ces différents concepts. Le premier sens de "récit émergent", tel qu'introduit par R. Aylett ${ }^{12}$, fait référence au concept d'émergence en intelligence artificielle: la création de complexité de manière ascendante, par l'interaction de nombreux éléments simples. Une intrigue émergente qui relèverait de ce principe serait un autre type d'intrigue résultante, ni contrôlée ni coïncidente. Si nous n'en parlons pas, c'est que nous considérons que sa possibilité théorique n'a pas été observée en pratique, si l'on prend effectivement l'émergence dans le sens d'un passage à la complexité. En effet, dans des applications fondées explicitement sur le concept d'émergence telles que FearNot ${ }^{13}$ ! ou the Virtual Storyteller ${ }^{14}$, d'une part les éléments simples sont des entités finalement complexes, comprenant parfois un volet personnage et un volet acteur ${ }^{15}$, et d'autre part les intrigues résultantes ne sont pas vraiment complexes. D'ailleurs, l'improvisation théâtrale, à laquelle les systèmes qui s'appuient sur le récit émergent se réfèrent souvent, ne relève pas non plus de l'émergence au sens de l'Intelligence Artificielle. Par contre, le sens second de "récit émergent ", celui d'une création de récit par le bas, sans structure narrative englobante de type structure aristotélicienne en trois actes mais sur la base de " process ", recouvre assez bien l'idée de l'intrigue contrôlée. Ainsi, des mécanismes narratifs de type « double appraisa ${ }^{16}{ }^{16}$ (sélection de l'action qui maximise l'impact émotionnel sur les autres agents) ou « late commitment ${ }^{17}$ » (spécification d'éléments du monde fictionnel du passé pour satisfaire des contraintes narrative présentes) sont à même de générer des intrigues résultantes contrôlées, puisque les événements produits sont directement explicables par celui ou celle qui connaît le système informatique (ce qui ne serait pas le cas dans le cas d'une émergence narrative au sens premier du terme). Quant au terme de "emergent gameplay ", il prend des sens assez variés, d'autant plus qu'il est utilisé aussi chez les joueurs eux-mêmes. Si le sens premier d'émergence se retrouve dans certains travaux théoriques sur les jeux vidéo ${ }^{18}$, le terme regroupe aussi les pratiques ludiques visant à exploiter les failles du jeu, les bugs, afin de créer des expériences nouvelles ${ }^{19}$. On pourrait parler à ce propos d'intrigues détournées. S'agissant d'un usage plus rare, elles supposent un volume d'utilisateurs assez importants, ce qui n'est pas le cas du récit interactif NFD. Pour Façade par contre, on trouve ce type de comportements, à en juger par quelques vidéos postées sur Internet ${ }^{20}$.

\section{(Re)définir la qualité d'une intrigue?}

Il est tentant de voir les récits interactifs comme extensions des récits linéaires classiques, et d'ainsi utiliser les critères et méthodes de ces derniers pour les évaluer. Nous considérons au contraire le récit interactif comme un media distinct, dans lequel la 
question de la qualité de l'intrigue doit être repensée. Deux arguments peuvent être avancés pour étayer cette thèse. D'une part, il apparaît difficile de demander aux algorithmes informatiques de produire des intrigues aussi bien ficelées que celles produites par les auteurs d'une fiction linéaire. Certes, des recherches existent en créativité computationnelle ${ }^{21}$, mais dans le cadre du récit, les résultats sont encore préliminaires. D'autre part, et de manière plus fondamentale, interagir avec un récit est une expérience différente de percevoir ce récit sans agir dessus, même si les événements qui se succèdent, l'intrigue, sont les mêmes ${ }^{22}$. Pour illustrer ce fait, s'il fallait retranscrire une séance de jeu de rôle ayant duré 4 heures par un film de la même durée, ce film susciterait un intérêt très limité, comparé à l'expérience vécue les joueurs. Le récit interactif est avant tout une expérience narrative, qui résulte du processus d'interaction ${ }^{23}$ . Comment alors définir la qualité d'une intrigue, dans le récit fortement interactif? Nous n'apporterons ici que quelques pistes, exploratoires, qui nous l'espérons, pourront susciter des études plus approfondies sur cette problématique largement inexplorée.

Une des manières de procéder consiste à adopter une démarche comparative: quelles qualités de l'intrigue classique se perdent dans l'intrigue interactive (approche négative) et, réciproquement, quelles qualités se gagnent (approche positive)? Nous commencerons par l'approche négative. De manière générale, les récits fortement interactifs ont tendance à perdre en tension narrative. L'intervention de l'utilisateur va insérer des événements incongrus, des sous-intrigues peu " efficaces ", qui font perdre le fil de l'histoire, c'est-à-dire qui gênent la perception de l'intrigue courante (qu'elle soit principale, secondaire, ou contrôlée). Certes, c'est bien le rôle du moteur narratif d'intégrer toute action de l'utilisateur dans le récit, mais d'une part nos algorithmes sont encore bien rudimentaires, et d'autre part on peut supposer qu'aucune solution algorithmique ne peut intégrer toute divergence de l'utilisateur, tant une intrigue classique, pour laquelle les conditions de réception sont pourtant largement maîtrisés, semble le résultat d'un « réglage au millimètre» de tous les paramètres du récit, réglage dans lequel peu de divergence semble vraiment possible sans sérieusement déséquilibrer l'intrigue. Ainsi, dans le cadre du récit fortement interactif, peut-être faut-il faire le deuil de l'intrigue parfaite, cristalline, auquel le récit classique nous a habitué, au profit d'une intrigue plus décousue, plus distendue.

Par ailleurs, à un niveau micro-narratif, celui des actes narratifs, nous avons observé sur NFD que certains actes narratifs classiques semblent moins pertinents dans le cadre interactif. Par exemple, dans un dialogue, les actes d'acceptation ou de refus de quêtes par le joueur apparaissent inintéressants pour le joueur, car ils donnent peu de feed-back, alors que dans le récit classique, il s'agit d'un moment clef de l'intrigue. Il en va de même pour les actes « condamner » et « féliciter » que nous avons implémentés, en référence à la notion de "sanctions " (récompense, châtiment) de l'analyse structurale du récit ${ }^{24}{ }^{25}$ : quand ils sont sélectionnés par l'utilisateur, ils donnent peu de feed-back et s'avèrent peu pertinents. Il semblerait donc que les constituants habituels de l'intrigue classique ne s'appliquent pas si aisément au récit interactif. Ainsi, aux niveaux global et local, un certain nombre de caractéristiques de l'intrigue classique semblent perdues, quand on entre dans le cadre du récit fortement interactif.

Poursuivons par l'approche positive. Si nous restons au niveau micro-narratif, nous avons pu observer sur NFD que certains actes qui ne figuraient pas au premier plan des schémas narratologiques classiques de Bremond ou Courtès avaient a contrario un impact fort dans le récit. Par exemple, l'acte que nous avons appelé «délégation» (une sorte de 
« faire faire »), qui consiste à demander à un personnage de réaliser une tâche plutôt que de la réaliser soi-même, s'est avéré intéressant, pour l'auteur et pour l'utilisateur. Ainsi, la pratique d'écriture et de jeu semble nous indiquer que l'intrigue du récit fortement interactif s'appuie sur un autre vocabulaire que l'intrique classique, ou tout du moins que les échelles d'importance des constituants de ce vocabulaire sont modifiées. Il convient donc d'approfondir la question positive susmentionnée, concernant les qualités nouvelles de l'intrigue interactive. On a beaucoup prôné le concept d'agency (voir plus haut) comme étant la caractéristique essentielle du récit interactif, mais ses liens avec le concept d'intrigue restent flous. Un premier point de vue consiste à considérer que cette agency est une caractéristique additionnelle et distincte de la notion d'intrigue. Elle viendrait donc compenser les faiblesses de cette dernière. Un joueur pourrait donc résumer son expérience ainsi : «l'intrigue n'était pas très bien ficelée, mais j'ai aimé influencer l'histoire». Un autre point de vue, que nous adoptons, consiste à repenser l'intrigue elle-même, étant donné son nouveau mode de consommation. Par l'action de l'utilisateur, une autre forme d'intrigue se dessine, dont les qualités sont à redéfinir. Sur le plan émotionnel, l'intrigue classique est fortement associée à la notion de suspense. Cette émotion doit-elle être remplacée ou complétée par d'autres émotions propres au récit interactif? Selon $\mathrm{C}$. Roth ${ }^{26}$ le suspense existe dans le récit interactif mais relève d'une émotion double: aux états psychologiques d'espoirs, craintes puis soulagement propres au suspense classique viennent s'ajouter les sentiments de fierté, de compétence et de satisfaction d'avoir réussi. Dans cette hypothèse, les émotions qui président l'intrigue interactive sont donc plus riches que pour son homologue non interactif.

Quand l'utilisateur échoue, c'est au contraire un sentiment de frustration qui est éprouvé, frustration de ne pas avoir atteint un objectif de l'histoire. Ce sentiment de frustration n'est en effet pas rare dans le récit interactif : il a souvent été mentionné lors de tests de $\mathrm{NFD}$, quand l'utilisateur se heurte aux comportements difficiles et imprévisibles du père. De même, Façade fonctionne beaucoup sur l'émotion de frustration ${ }^{27}$, d'autant plus que le but implicite du jeu, réconcilier le couple, est rarement atteint. Cette idée de frustration comme émotion propre au récit interactif est peut-être à rapprocher de l'Esthétique de la frustration, approche proposée par P. Bootz pour caractériser certaines œuvres numériques qui mettent en scène l'échec de l'interaction, cet échec lui-même portant le sens de l'œuvre ${ }^{28}$.

En mode interactif, l'intrigue réussie sera donc celle qui saura au mieux gérer les sentiments de compétence et de frustration. Il est intéressant à ce niveau de faire un parallèle avec les études sur l'apprentissage et les jeux pédagogiques, qui traitent aussi ces deux sentiments. Dans la théorie du Flow notamment, souvent reprise dans le domaine des jeux vidéo d'apprentissage, un apprentissage optimal consiste à doser la difficulté des tâches à réaliser, afin que de mettre l'apprenant en position de challenge, mais pas en situation d'ennui (tâche trop facile) ni de frustration (tâche trop difficile ${ }^{29}$ ). Même si la situation en récit interactif est différente, puisque la frustration peut être un but en soi, ce parallèle nous permet de préciser ce que sont les sentiments de compétence et de frustration dans le cas du récit interactif. La satisfaction de réussir ne doit pas s'assimiler à la joie de recevoir un cadeau gratuit (tâche facile); la frustration suite à l'échec ne doit pas être confondue avec le sentiment de rejet d'être confronté à une tâche visiblement inaccessible. Par le dosage de la difficulté, l'intrigue interactive doit maintenir l'utilisateur dans un état d'incertitude accompagnée de frustration ou de satisfactions intermédiaires, pour se conclure par un état final polarisé vers l'un de ces 
deux pôles. Ce rôle déterminant de la difficulté des problèmes posés à l'utilisateur n'est pas surprenant si l'on considère le récit interactif comme une forme de jeu - mais c'est un postulat que nous ne souhaitions pas adopter a priori. En effet, le dosage de la difficulté est une des clefs du game design ${ }^{30}{ }^{31}$. Dans le cas du récit fortement interactif, la compétence concernée n'est ni sensori-motrice comme dans les jeux d'action, ni logicomathématique comme dans les jeux à énigmes. Elle serait plutôt socio-psychologique, car le récit, par définition, met en œuvre des personnages dans leur quête et leur transformation ${ }^{32} 33$. Construire une bonne intrigue interactive nécessiterait donc aussi de programmer des mécaniques socio-psychologiques à même d'impliquer le joueur dans des tâches ou missions difficiles mais qu'il peut maîtriser.

La frustration quant à elle n'est en général pas une expérience d'utilisation particulièrement recherchée. Pourtant, il semble, à travers les quelques exemples à notre disposition, qu'il s'agisse d'une émotion fondamentale du récit fortement interactif. Mieux que toute autre forme narrative, le récit interactif est à même de faire ressentir la frustration de nombres de situations humaines. Alors que le récit classique nous fait ressentir ce type d'émotions par empathie, l'action sur le récit, si elle détruit cette empathie car cette dernière est fondamentalement liée à l'inaction du spectateur, fait vivre réellement l'émotion de frustration.

Pour résumer, deux émotions, la satisfaction de réussir et la frustration semblent être constitutives du récit interactif et définissent ainsi la qualité du récit interactif selon des critères propres, c'est-à-dire distincts du récit linéaire classique. Ces deux émotions sont le pendant des deux issues possibles de l'intrigue classique : soit le protagoniste réussit, soit le protagoniste échoue. Comme le protagoniste ${ }^{34}$ est contrôlé par le spectateur devenu utilisateur/joueur, l'échec ou le succès du protagoniste est aussi celui du joueur. Il n'y a pas pour autant totale fusion des deux entités, puisque quand le héros souffre, meurt, l'utilisateur reste indemne. A ce propos, Marie-Laure Ryan notait qu'un spectateur actif ne souhaiterait pas jouer un personnage tragique, car personne n'aimerait souffrir comme ces personnages. A la lumière de notre discussion, il semblerait que ces personnages pourraient être joués par un utilisateur, s'il est donné à ces derniers une possibilité perçue comme réelle de sauver ces personnages tragiques en agissant à leur place, même si ces efforts devaient s'avérer infructueux (émotion de frustration).

\section{Conclusion}

Par une approche avant tout empirique, en ce qu'elle s'appuie sur quelques œuvres existantes, nous avons ébauché une typologie des intrigues dans le récit fortement interactif, et proposé de considérer la qualité des intrigues de ce nouveau mode narratif selon des critères distincts et spécifiques. Deux émotions s'avèrent ainsi constitutives du récit fortement interactif : la satisfaction de réussir et la frustration.

Pour aller plus loin, sur le plan empirique pour commencer, il apparaît d'une part nécessaire de disposer d'un corpus plus important, afin d'éviter de tirer des conclusions trop générales qui ne seraient dues qu'aux spécificités d'un genre naissant. D'autre part, une approche plus expérimentale pourrait être envisagée, visant non seulement à vérifier les hypothèses présentées dans le présent article mais aussi à sonder, de manière plus ouverte, les éléments de l'intrigue particulièrement prégnants pour le récit interactif. Sur le plan théorique, il y a certainement des rapprochements à faire avec les "game studies", qui depuis environ quinze ans ont pris le jeu vidéo comme objet d'étude. 
Cependant, sans revenir à l'opposition caricaturale entre jeu et récit qui a accompagné la naissance des "game studies ", nous ne souhaitons pas assimiler jeu et récit, notamment en ce qui concerne la notion de gain - le fait de gagner ou de perdre - qui convient mal au récit interactif. Les exemples mettant en jeu le sentiment de frustration en sont une parfaite illustration.

\section{NOTES}

1. Brenda Laurel, "Towards the Design of a Computer-Based Interactive Fantasy System" (Ohio State University, 1986).

2. Sean Smith and Joseph Bates, Towards a Theory of Narrative for Interactive Fiction - CMUCS-89-121, 1989.

3. Janet Horowitz Murray, Hamlet on the Holodeck: The Future of Narrative in Cyberspace, Free Press (New York: Free Press, 1997).

4. Jean-Noël Portugal, "L'avenir Dira Ce Que Nous Aurons Créé », in Création de récits pour les fictions interactives: simulation et réalisation, ed. by Nicolas Szilas and Jean-Hugues Réty (Paris: Hermes \& Lavoisier, 2006), pp. 133-165.

5. http://www.interactivestory.net

6. http://www.versu.com

7. http://tbisim.unige.ch/portal

8. Michael Mateas and Andrew Stern, "Build It to Understand It : Ludology Meets Narratology in Game Design Space”, in DiGRA Conference, 2005, ii.

9. Nicolas Szilas, Olivier Marty and Jean-hugues Réty, "Authoring Highly Generative Interactive Drama," in Virtual Storytelling, Second International Conference (ICVS 2003). LNCS 2897, ed. by Olivier Balet, Gérard Subsol, and Patrice Torguet (Heidelberg: Springer Verlag, 2003), pp. 37-46.

10. Linda Seger, Making a Good Script Great (Hollywood, CA: Samuel French Trade, 1989).

11. Mark Riedl, C J Saretto and $R$ Michael Young, "Managing Interaction Between Users and Agents in a Multi-Agent Storytelling Environment", in Proceedings of the Second International Joint Conference on Autonomous Agents and Multiagent Systems, AAMAS '03 (New York, NY, USA: ACM, 2003), pp. 741-48.

12. Ruth Aylett, "Narrative in Virtual Environments - Towards Emergent Narrative", in Proceedings of the AAAI fall symposium on Narrative Intelligence, ed. by Michael Mateas and Phoebe Sengers, Working Note of the Narrative Intelligence Symposium (The AAAI Press, 1999), pp. 83-86

http://www.aaai.org/Papers/Symposia/Fall/1999/FS-99-01/FS99-01-014.pdf.

13. R. S. Aylett and others, "FearNot ! - an Experiment in Emergent Narrative," in Intelligent Virtual Agents, ed. by Themis Panayiotopoulos and others, Lecture Notes in Computer Science (Heidelberg: Springer, 2005), mmmdclxi, 305-16.

14. Ivo Swartjes and Mariët Theune, "The Virtual Storyteller: Story Generation by Simulation," in BNAIC, ed. by Anton Nijholt and others (Enschede, The Netherlands: University of Twente, 2008), pp. 257-64

http://wwwhome.cs.utwente.nl/ theune/PUBS/bnaic08-Swartjes-Theune.pdf. 
15. Sandy Louchart, Ruth Aylett and Joao Dias, "Double Appraisal for Synthetic Characters," in Intelligent Virtual Agents, ed. by Catherine Pelachaud and others, Lecture Notes in Computer Science (Heidelberg: Springer, 2007), mmmmdccxxii, 393-94.

16. Louchart, Aylett and Dias, mmmmdccxxii.

17. Ivo Swartjes, Edze Kruizinga and Mariët Theune, "Let's Pretend I Had a Sword: Late Commitment in Emergent Narrative", in First Joint International Conference on Interactive Digital Storytelling (ICIDS). LNCS, 5334, ed. by Ulrike Spierling and Nicolas Szilas, LNCS (Berlin / Heidelberg: Springer, 2008), mmmmmcccxxxiv, 264-67.

18. Katie Salen and Eric Zimmerman, Rules of Play: Game Design Fundamentals (MIT press, 2004).

19. Prévieux Julien, « Jouer Avec Le Jeu - Erreur, Errance et Émergence », artpress2, 2013, 18-24.

20. Sur Youtube, on trouve ainsi plusieurs détournements de plus ou moins bon goût de Façade : https://www.youtube.com/watch?v=9bpCL-eUsDU, https://www.youtube.com/watch?v=pKbAKg8KMQ, https://www.youtube.com/watch?v=17xjE8dDtT4, etc.

21. Pablo Gervás, "Computational Approaches to Storytelling and Creativity," AI Magazine, 30 (2009), 49-62.

22. Nicolas Szilas, "Requirements for Computational Models of Interactive Narrative," in Computational Models of Narrative, papers from the 2010 AAAI fall symposium, ed. by Mark Finlayson (Menlo Park, California: AAAI Press, 2010), pp. 62-68.

23. Ruth Aylett and Sandy Louchart, "Being There: Participants and Spectators in Interactive Narrative," in Fourth International Conference on Virtual Storytelling (ICVS 2007). LNCS 4871, ed. by Marc Cavazza and Stéphane Donikian, LNCS (Heidelberg: Springer, 2007), mmmmdccclxxi, 117-28.

24. Claude Bremond, Logique Du Récit (Paris: Seuil, 1973).

25. Joseph Courtès, Analyse Sémiotique Du Discours, Paris, Hachette, Hachette (Paris, 1991).

26. http://tecfalabs.unige.ch/mediawiki-narrative/index.php/Suspense

27. Rachel Lee Knickmeyer and Michael Mateas, "Preliminary Evaluation of the Interactive Drama Facade," in CHI 05 extended abstracts on Human factors in computing systems (New York, NY, USA: ACM, 2005), pp. 1549-52.

28. Philippe Bootz, «L'oeuvre Signe, Texto Digital », 3 (2007).

29. Thomas $\mathrm{W}$ Malone and Lepper Mark R, "Making Learning Fun: A Taxonomy of Intrinsic Motivations for Learning," in Aptitude, Learning, and Instruction, III: Cognitive and Affective Process Analysis, ed. by R.E. Snow and M.J. Farr (Hillsdale, NJ: Lawrence Erlbaum Associates, 1987), pp. 223-53.

30. Bob Bates and LaMothe André, Game Design: The Art \& Business of Creating Games (Roseville, CA: Prima Tech, 2001).

31. Salen and Zimmerman.

32. Jean-Michel Adam, Le Texte Narratif (Paris: Nathan, 1994).

33. Marie-Laure Ryan, "Introduction," in Narrative Across Media, ed. by Marie-Laure Ryan (Lincoln and London: University of Nebraska Press, 2004).

34. Ed Tan, Emotion and the Structure of Narrative Film. Film as an Emotion Machine (Mahwah, NJ: Erlbaum, 1996). 


\section{ABSTRACTS}

This article focuses on highly interactive narrative, an emerging genre which has concentrated advanced research in computing since the early nineties. The goal of these researches - if not their "Grail" - is to produce narratives in which the audience determines by their actions the course of events in the story. Much has been written regarding the potentialities of such a vision, in terms of how the status of the plot is transformed and how the audience, the story, the machine and the author interrelate. But what happens at the concrete level of produced works? From a few examples of such narratives, including Nothing For Dinner that has been developed in our laboratory, we propose a typology of plots in highly interactive narratives. In addition, we defend the thesis that the plot in highly interactive narrative is fundamentally different from the classical plot, and we suggest some directions to characterize this particular plot.

Nous nous intéresserons dans cette contribution au récit fortement interactif, qui fait l'objet de recherches informatiques depuis les années 1990. L'objectif de ces recherches, pour ne pas dire l'idéal, est de produire des récits dans lesquels le spectateur contrôle fortement le déroulement de l'histoire. Il a beaucoup été écrit sur les potentialités d'un tel programme, notamment en terme de bouleversement de l'intrigue et du rapport entre le spectateur, l'histoire, la machine et l'auteur. Mais qu'en est-il en réalité, dans le concret des récits fortement interactifs ? A partir de quelques rares exemples de tels récits, dont Nothing For Dinner développé dans notre laboratoire, nous proposons d'une part une typologie des intrigues rencontrées dans ce type de récits numériques. D'autre part, nous avançons la thèse que l'intrigue dans le récit interactif est distincte de son homologue linéaire, et dégageons quelques pistes pour la caractériser.

\section{INDEX}

Chronological index: XXIème siècle

Mots-clés: récit interactif, récit fortement interactif, intrigue, écriture interactive, compétence, frustration

\section{AUTHOR}

\section{NICOLAS SZILAS}

TECFA-FPSE, Université de Genève, nicolas.szilas@unige.ch

Nicolas Szilas est Maître d'Enseignement et de Recherche au laboratoire TECFA de l'Université de Genève, spécialisé dans les technologies éducatives. Il étudie le récit interactif depuis de nombreuses années, au travers notamment de la réalisation d'un système informatique nommé IDtension. Il s'intéresse particulièrement à l'utilisation du récit interactif à des fins d'apprentissage ou de formation, au développement de modèles, outils et méthodologies permettant à des auteurs de s'approprier ces nouvelles technologies d'écriture, et enfin à l'exploration de nouvelles formes expressives s'appuyant sur la narration interactive. Il 
s'intéresse également de près aux jeux vidéo pédagogiques, domaine qu'il enseigne à l'Université, notamment aux questions d'intégration des objectifs pédagogiques dans la mécanique de jeu. 